Classification

Physics Abstracts

$81.15 \mathrm{H}-47.70 \mathrm{H}$

\title{
Chemical Vapor Deposition of Thick Tungsten Coatings: Mass Transport Modelling and Experiments
}

\author{
M. Pons $\left({ }^{1}\right)$, A. Benezech $\left({ }^{1}\right)$, P. Huguet $\left({ }^{2}\right)$, R. Gaufres $\left({ }^{2}\right)$, Ph. Diez $\left({ }^{3}\right)$ and D. Lafforet $\left({ }^{3}\right)$ \\ (1) Laboratoire Science des Surfaces et Matériaux Carbonés, URA CNRS nº 413 - ENSEEG - \\ INPG, BP 75 Domaine Universitaire, 38402 Saint-Martin d'Hères, France \\ $\left({ }^{2}\right)$ Laboratoire de Spectrométrie Moléculaire, USTL - Place Eugène Bataillon, 34095 Montpel- \\ lier, France \\ $\left({ }^{3}\right)$ COMURHEX, BP 29, 26701 Pierrelatte, France
}

(Received 6 October 1994, revised 10 April 1995, accepted 16 may 1995)

\begin{abstract}
Thick tungsten coatings have been produced by chemical vapor deposition (CVD) from $\mathrm{H}_{2}-\mathrm{WF}_{6}$ at a temperature in the range $773-1073 \mathrm{~K}$ under a reduced pressure. The experimental set-up was designed for in situ Raman analysis of the gas phase (temperature and $\mathrm{WF}_{6}$ concentration) during the growth of tungsten coatings. A two dimensional mass transport model was proposed. It assumes a simple chemical pathway. Only the $\mathrm{H}_{2}$ reduction of $\mathrm{WF}_{6}$ on the substrate was taken into account. The major objective of the present paper is to report on (i) the experimental deposition rates and the rate values calculated by the model, (ii) the values of temperature and gas phase composition deduced from Raman spectroscopy measurements and the values of these quantities obtained by numerical calculations. Within the range of process parameters investigated, the predictive capabilities of the numerical modelling are demonstrated; in addition the temperature and $\mathrm{WF}_{6}$ partial pressure measurements can be recorded by a Raman equipment during the deposition process.
\end{abstract}

\section{Introduction}

In recent years, a considerable interest was devoted to chemical vapor deposition (CVD) of tungsten films for microelectronic applications [1-7]. Tungsten is a suitable metal to produce interconnect lines in microelectronics devices owing to its relatively low resistivity $(5.2 \mu \Omega \mathrm{cm})$, good electromigration resistance and its thermal expansion coefficient similar to that of silicon. Up to $5 \mathrm{~mm}$ thick tungsten coatings have been produced by the hydrogen reduction of $\mathrm{WF}_{6}$ under various operating conditions (temperatures, partial pressures, flow rates, reactor geometry...) [8]:

$$
\mathrm{WF}_{6}+3 \mathrm{H}_{2} \longrightarrow \mathrm{W}+6 \mathrm{HF}
$$

Recently, numerical models for CVD process of tungsten films, at a temperature in the range 600-800 $\mathrm{K}$ under a reduced total pressure varying from 100 to $1000 \mathrm{~Pa}$ and for $\mathrm{WF}_{6}$ dilution 
from 0.1 to $6 \mathrm{~mol} \%$, based upon some combination of heat, mass and momentum transfer were presented [9-16]. In these studies the deposition rate of tungsten films was assumed to be controlled by a surface reaction step.

Different rate-controlling steps for the surface reaction were proposed, namely the dissociative adsorption of hydrogen, the surface reaction between hydrogen and fluorine adatoms or the desorption of hydrogen fluoride [1-7]. Recently, the growth kinetics of tungsten films produced by CVD was re-examined. The HF desorption may be the rate-limiting step as the reaction order with respect to the hydrogen pressure is equal to 0.5 [2-5]. The phenomenological form of the rate law generally assumed for reaction (1) is:

$$
\text { Rate } \propto P_{\mathrm{WF}_{6}}^{0} P_{\mathrm{H}_{2}}^{0.5} \exp \left(-\frac{E}{R T}\right)
$$

where $\mathrm{P}_{\mathrm{WF}_{6}}$ and $\mathrm{P}_{\mathrm{H}_{2}}$ are the reactant partial pressures and $E$ the apparent activation energy (about $66 \mathrm{~kJ} \mathrm{~mol}^{-1}$ ). More details concerning the surface reaction mechanisms can be found in recent studies $[4,5]$.

By linking the conservation equations to kinetic data, modelling studies have been performed to optimize the process and the equipment $[9,15]$. Industrial equipment for selective tungsten deposition have been optimized by accurate modelling studies including not only the main reactive species $\left(\mathrm{H}_{2}, \mathrm{WF}_{6}\right.$ and $\left.\mathrm{HF}\right)$ but also reactive intermediates $\mathrm{WF}_{x}$ and $\mathrm{SiF}_{y}$ which are considered responsible for selectivity loss [15]. The calculations have shown that it was possible to reduce the HF concentration near the substrate surface by changes in the reactor design.

When moving towards a higher temperature range $(773 \mathrm{~K}-1073 \mathrm{~K})$ and a higher pressure range (1000 to $4000 \mathrm{~Pa}$ ), even using higher $W_{6}$ partial pressures, the growth kinetics of tungsten films can become mass-transport controlled. The major purpose of the present work is to delimit the different rate-limiting regimes in the $773 \mathrm{~K}-1073 \mathrm{~K}$ temperature range from the deposition rate of tungsten films, mass transport modelling and $\imath n$-situ Raman diagnostics of the gas phase (temperature and $\mathrm{WF}_{6}$ concentration). This last technique was used by Fotiadis et al. [17] to measure the inert gas temperature in a CVD reactor. Under the experimental conditions (temperature and pressure) investigated, Raman scattering was observed to be very weak. The measurements from spectral signals originating from spontaneous Raman effect are difficult to perform. These limitations can be overcome using either non-linear techniques, such as Coherent Anti-Stokes Raman Scattering (C.A.R.S.) [18] or the equipment developed in this work in which spontaneous Raman permits to record a fair spectrum of the vapor of a good scatterer $\left(\mathrm{C}_{10} \mathrm{H}_{8}\right)$ under a pressure of $11.5 \mathrm{~Pa}$ at room temperature. Having in view this study, we also discussed and improved the reliability of Raman intensity measurements, from which the temperature and the composition of the gas phase are determined, and developed new methods.

\section{Raman Measurements}

2.1. The Raman Equipment. - This equipment was already described in detail $[19,20]$. The excitation of the spontaneous Raman spectrum was obtained by placing the reactor inside the modified cavity of a commercial argon laser (Highlight of Coherent Radiation, power output warranted $4 \mathrm{~W}$ all lines). The front mirror of the laser was removed and the resonant cavity was completed by a lens $(f=0.20 \mathrm{~m})$ and a spherical mirror $(R=0.20 \mathrm{~m})$ of maximum reflectance for $\lambda=500 \mathrm{~nm}$ (Fig. 1). Using the intra-cavity configuration the output laser power could not be determined since no light was emitted by the system; however, the radiation density exciting the gas phase was about 50 times higher than the density obtained with the 


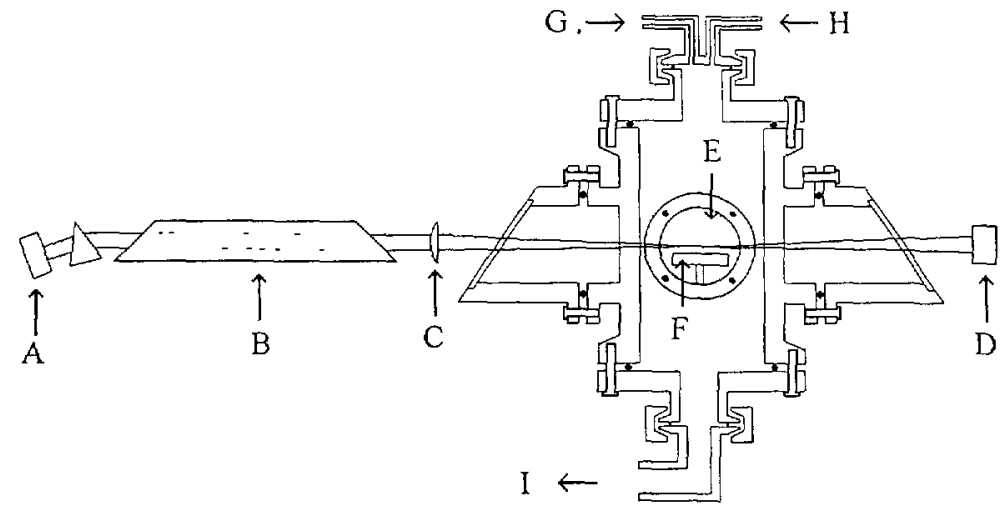

a)

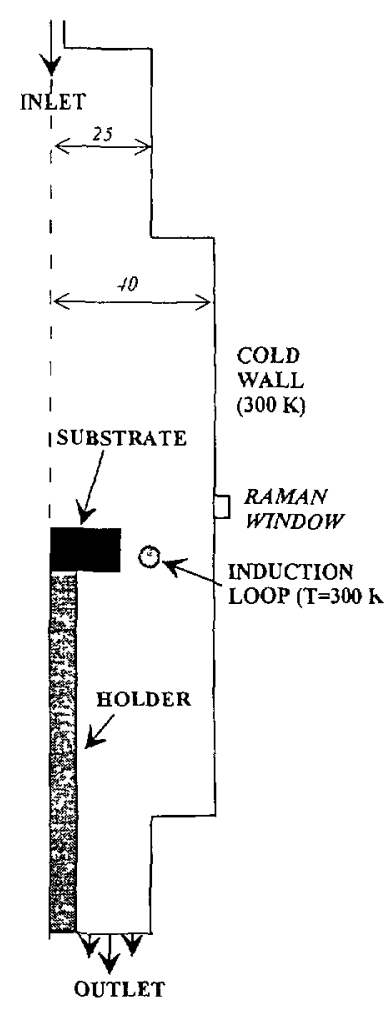

b)

Fig. 1. - a) Cross section of the reactor: A - Plane mirror. B - Laser tube. C - Lens $f=0.20 \mathrm{~m}$. D - Spherical mirror $R=0.20 \mathrm{~m} . \mathrm{E}-\mathrm{Ca} \mathrm{F}_{2}$ windows. $\mathrm{F}$ - Graphite substrate. $\mathrm{G}$ - Gas mixture inlet. $\mathrm{H}$ - Pressure measurement. I - Exit. b) Schematic representation for modelling studies (dimensions are in $\mathrm{mm}$ ).

conventional laser configuration. The laser was operated in the longitudinal mode to produce the highest radiation density. In addition, a diaphragm was placed in the cavity to obtain a pure transversal mode $\left(\mathrm{TEM}_{00}\right)$. The blue line $(489 \mathrm{~nm})$ was preferred to the green one for such an intra-cavity excitation, since the density of the radiation obtained was less sensitive to a possible absorption inside the cavity.

The intensified multi-channel OMARS 89 spectrometer from Dilor (France) was equipped with a dispersive system of a $0.32 \mathrm{~m}$ focal length with a grating of 1800 grooves $\mathrm{mm}^{-1}$ The geometrical slitwidth was typically $200 \mu \mathrm{m}$, corresponding to a spectral bandwidth of $8 \mathrm{~cm}^{-1}$. The beams of the scattered light entering the spectrometer and falling on the diode array of the multi-channel detection originated from a gas volume whose cross-section was a rectangle of $(280 \times 57) \mu \mathrm{m}^{2}$. This defines the spatial resolution achieved in the experiments.

2.2. The CVD REACTOR. - The experimental CVD reactor, equipped with three optical windows for the excitation and observation of the Raman scattering, was a scaled model of an industrial CVD reactor (Fig. 1). The CVD chamber was made of stainless steel, with a watercooled jacket. The Brewsterian windows for the laser beam were placed at the end of muffs as 
long as possible, in order to prevent any deposition of reaction products on fluorite windows. Theses muffs were also water-cooled. The graphite substrate (disc of $33 \mathrm{~mm}$ in diameter and $10 \mathrm{~mm}$ in height), was inductively heated and its temperature was measured by means of a thermocouple. The maximum output power of the high frequency generator was $3 \mathrm{~kW}$ and 50 to $80 \%$ of the maximum power was allowed in the loop according to the required temperature. Insofar as the reaction had reached a steady rate, the temperature remained fairly constant for several hours. The flow rates of reactant gases $\left(\mathrm{H}_{2}\right.$ and $\left.\mathrm{WF}_{6}\right)$ were controlled by mass flowmeters. Since the flow proceeded downstream, the deposition of tungsten occurred with velocity, temperature and species concentration fields interacting among themselves through mass transport and chemical reactions. The laser beam was focused just above the substrate surface along the axis of the reactor. In order to vary the distance of the studied point from the substrate surface, the substrate holder was vertically moved by a micrometer screw, all the other parts of the reactor and the laser beam remaining fixed.

2.3. Raman Intensity MEasurements. - The temperature and composition of the gas phase were determined by Raman intensity measurements, or, more precisely, calculated from the intensity ratio of two Raman lines, the intensity of the Raman signal (line or band) being defined by its area. Experiments were designed to investigate (i) the stability of the intensity ratio of two Raman lines over a one day experiment, with a given optical and spectral adjustment and (ii) the reproducibility of an intensity ratio after a complete resetting of the optical and spectral adjustments [19]. In typical experiments, 100 recordings were made over one day and the intensity ratio $I_{J=2} / I_{J=1}$ of the rotation lines of hydrogen due to the transitions starting from the rotational levels $J=2$ and $J=1$ respectively was measured. For the stability, we found a standard deviation of $0.025 \%$ over one day, corresponding, at room temperature, to a standard deviation of $0.06 \mathrm{~K}$. For the reproducibility, we obtained deviations of about $0.15 \%$ from one adjustment to another, corresponding, at room temperature, to temperature deviations of $0.4 \mathrm{~K}$.

2.4. Temperature Measurements. - The temperature in the gas phase was independently determined from the spectral signals of hydrogen and tungsten hexafluoride, by two different methods. Both methods directly or indirectly based on the Boltzmann's law corresponded to quite different experimental approaches.

For hydrogen, we used the temperature dependence of the intensity ratio of two rotational lines, according to the following equation [20]:

$$
1 / T=\left(1 / T_{\text {cal }}\right)-(k / h c)\left[F\left(J_{2}\right)-F\left(J_{1}\right)\right]^{-1} \operatorname{Ln}\left[\left(I_{2 T} / I_{1 T}\right) /\left(I_{2 \text { cal }} / I_{1 \mathrm{cal}}\right)\right]
$$

in which:

$T_{\text {cal }}$ is a calibration temperature (typically $298 \mathrm{~K}$ ), and $T$ the temperature to be determined, $F\left(J_{2}\right)$ and $F\left(J_{1}\right)$ are the rotational spectral terms corresponding to the rotational quantum numbers $J_{2}$ and $J_{1}$ respectively, $I_{2}$ and $I_{1}$ are the apparent intensities of the rotational lines corresponding to the transitions starting from the levels $J_{2}$ and $J_{1}$ respectively (in most experiments, $J_{1}=1$ and $J_{2}=2$ ). The further subscript, $T$ or cal, refers to the temperature. $k$, $h$ and $c$ are Boltzmann, Planck and light velocity constants.

The temperature of tungsten hexafluoride was determined from the alteration of the profile of the $\nu_{1}$ band $\left(772 \mathrm{~cm}^{-1}\right)$ which is temperature dependent, according to analytical expressions in the most favourable cases, and that can be used for temperature determinations [21-23]. During this work we have developed a more empirical method [24], based on the alteration of a profile which may be very accurate. 


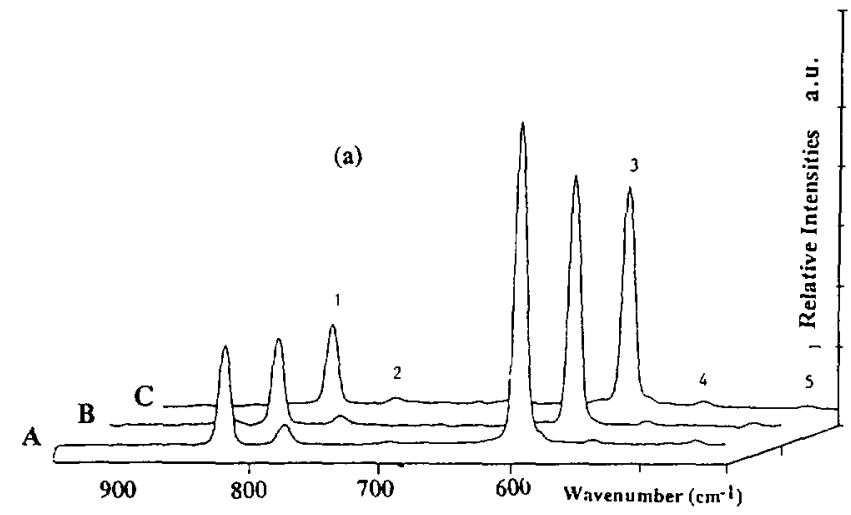

a)

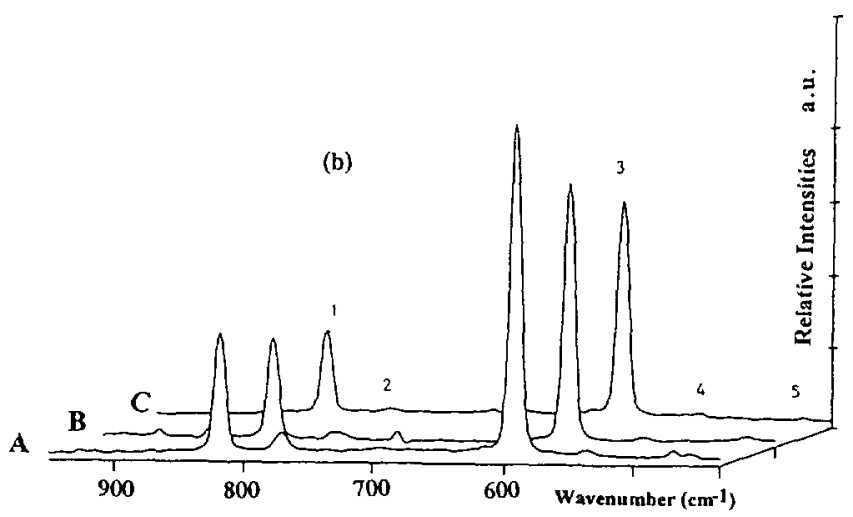

b)

Fig. 2. - 3-D representation of Raman spectra of the reactive mixtures at various distances from the substrate $(10,5,2.5 \mathrm{~mm})$ and for different substrate temperatures. The common parameters for both experiments are: $P=4000 \mathrm{~Pa}$; initial mole fraction ratio of $\mathrm{WF}_{6}: 4 \mathrm{~mol} \%$; inlet flow rate $=200$ sccm (STP). Distance from the substrate: (A) $10 \mathrm{~mm}$, (B) $5 \mathrm{~mm}$, (C) $2.5 \mathrm{~mm}$. a) $-T_{\text {subst. }}=773 \mathrm{~K}$. b) $-T_{\text {subst }}=923 \mathrm{~K}$. Raman signals. 1 and 3: rotational lines of $\mathrm{H}_{2} .2: \nu_{1}$ of $\mathrm{WF}_{6}, 4$ and 5: rotational lines of HF.

The three Raman signals used for temperature determination, namely the two rotational lines of hydrogen at $\Delta \tilde{\nu}=586.7$ and $813.4 \mathrm{~cm}^{-1}$ and the $\nu_{1}$ band of tungsten hexafluoride at $\Delta \tilde{\nu}=772 \mathrm{~cm}^{-1}$, were simultaneously recorded with our multichannel spectrometer for a given point of the gas phase, and were well separated (Fig. 2). The temperature values obtained from each chemical species were found to be somewhat different (Table I); various reasons can be invoked to explain these deviations:

(i) the temperatures were obtained by two different methods; with the method used for tungsten hexafluoride, the noise of the recording obviously resulted in an error by excess,

(ii) with a gas stream direction normal to the substrate surface, the gas mixture was out of thermal equilibrium, especially near the substrate,

(iii) tungsten hexafluoride absorbs infrared wavelength (and not hydrogen). 
Table I. - Examples of results obtained by Raman diagnostics. (experimental conditions of Fig. 3).

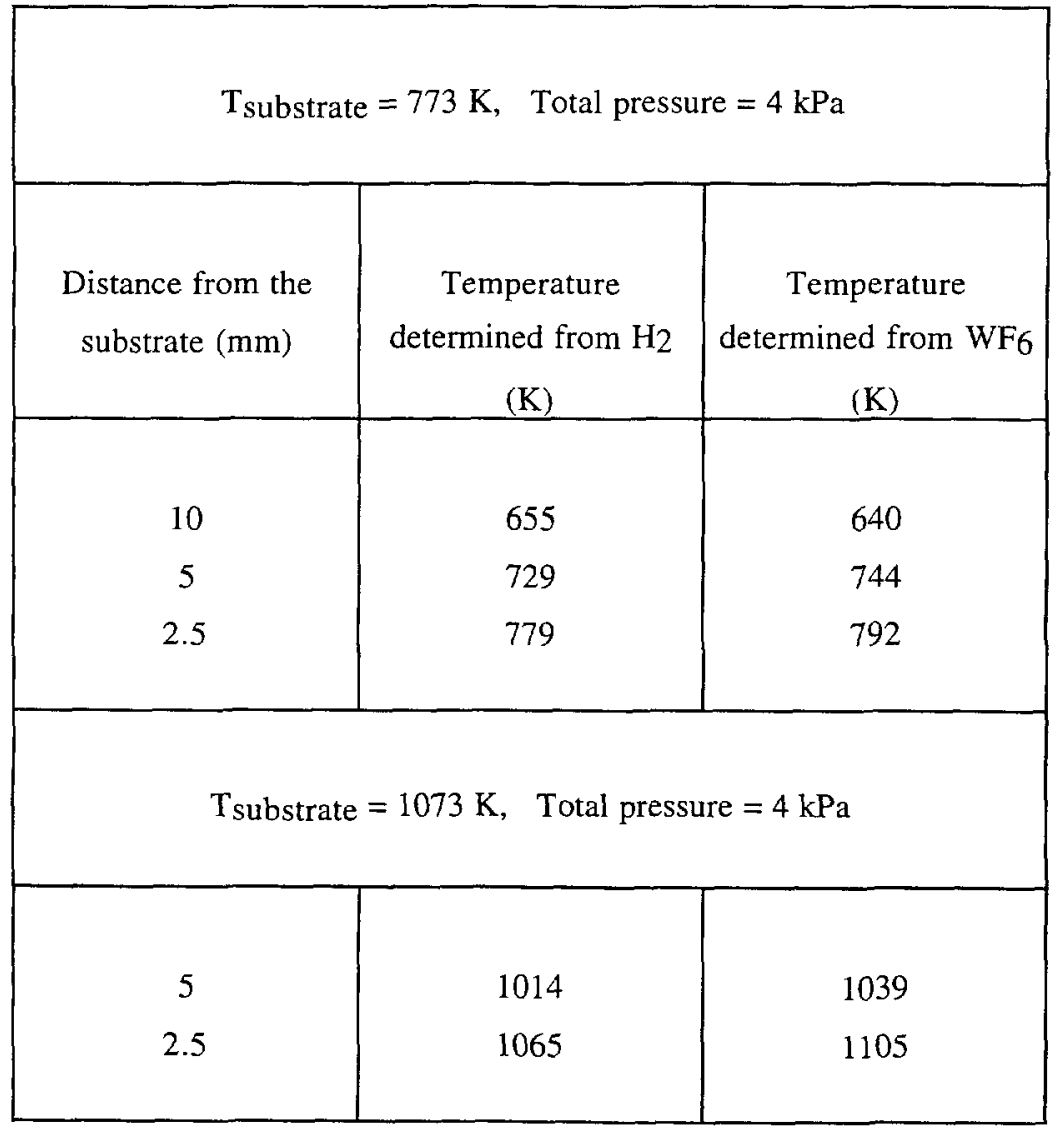

For the comparison with the modelling studies, we retained a molar fraction weighed average temperature, according to: $\bar{T}=x_{\mathrm{WF}_{6}} T_{\mathrm{WF}_{6}}+x_{\mathrm{H}_{2}} T_{\mathrm{H}_{2}}$ where $x_{\mathrm{WF}_{6}}$ and $x_{\mathrm{H}_{2}}$ are the molar fractions of $\mathrm{WF}_{6}$ and $\mathrm{H}_{2}, \mathrm{~T}_{\mathrm{WF}_{6}}$ and $\mathrm{T}_{\mathrm{H}_{2}}$ the temperatures of $W \mathrm{~F}_{6}$ and $\mathrm{H}_{2}$ respectively.

2.5. Chemical Composition Measurement. - The Raman signals of HF (its unique vibrational band and the rotation lines) were very weak. As a result, the temperature and the mole fraction of HF could not be accurately determined. For validation of the modelling, only the mole fraction ratios $x_{\mathrm{WF}_{6}} / x_{\mathrm{H}_{2}}$ were determined from the ratio of the Raman intensity of the $\nu_{1}$ band of tungsten hexafluoride to the intensity of the strongest rotational line of hydrogen, issued from the $J=1$ level. A calibration was performed from a mixture of known composition, at a room temperature out of the range of the reaction between the two species. In the reactive gas phase, the measured intensity ratio must be corrected from temperature dependence, which is not the same for both Raman signals, according to the following equation:

$$
\begin{aligned}
\left(x_{\mathrm{WF}_{6}} / x_{\mathrm{H}_{2}}\right)_{T} & =\left(x_{\mathrm{WF}_{6}} / x_{\mathrm{H}_{2}}\right)_{\mathrm{cal}}\left\{\left(I_{\mathrm{WF}_{6}} / I_{\mathrm{H}_{2}}\right)_{T} /\left(I_{\mathrm{WF}_{6}} / I_{\mathrm{H}_{2}}\right)_{\mathrm{cal}}\right\} \\
& \times\left\{\left(1-\exp \left[-\theta_{\mathrm{vib}} / T\right]\right) /\left(1-\exp \left[-\theta_{\text {vib }} / T_{\text {cal }}\right]\right)\right\} \\
& \times\left(Z_{\text {rot }}, \mathrm{H}_{2}, T_{\text {cal }} / Z_{\text {rot }} \mathrm{H}_{2}, T\right)\left(\exp [-F(1) h c / k T] / \exp \left[-F(1) h c / k T_{\text {cal }}\right]\right)
\end{aligned}
$$


in which $x_{\mathrm{WF}_{0}} / x_{\mathrm{H}_{6}}$ is the ratio of the mole fraction of tungsten hexafluoride to the mole fraction of hydrogen, $I_{\mathrm{WF}_{\mathfrak{G}}} / I_{\mathrm{H}_{2}}$ is the intensity ratio of the $\nu_{1}$ band of tungsten hexafluoride to the intensity of the $(J=1 \rightarrow J=3)$ rotational line of hydrogen, $\theta_{\text {vib }}$ is the vibrational characteristic temperature corresponding to the $\nu_{1}$ mode of tungsten hexafluoride, $Z_{\mathrm{rot} \mathrm{H}_{2}}$ is the partition function of rotation of hydrogen (which must be calculated as a sum and not as an integral) and $F(1)$ is the rotational spectral term of hydrogen for $J=1$.

The subscript "cal" refers to the calibration experiment, $T$ as a variable or a subscript refers to the temperature at which molar fraction ratio is determined.

Two series of typical Raman spectra are given in a $3 \mathrm{D}$ representation in Figure 2, each $3 \mathrm{D}$ series corresponds to an experiment. The three spectra in Figure 2 were obtained from measurements performed with different distances between the substrate surface and the laser beam. All the experimental parameters were maintained constant, except the temperature of the substrate. In each series, the temperature evolution was characterized by the evolution of the intensity ratio of the rotational lines of hydrogen and the consumption of tungsten hexafluoride was deduced from the decrease in intensity of its $\nu_{1}$ Raman peak.

\section{Modelling of Tungsten Deposition}

The model is based on multicomponent mass transport and only the surface reaction (1) (no homogeneous reaction) was considered.

3.1. General Equations. - The meaning of symbols used in various equations is given in Appendix A.

The two-dimensional hydrodynamic model for the gas flow, described by equations (B.1) and (B.2), Appendix B, is based on the following assumptions, namely steady-state conditions, laminar gas flow and the validity of the ideal gas law for the evaluation of density variations.

The energy expression given by equation (B.3) (Appendix B) is established assuming that: the dissipation and pressure terms as well as the emission and absorption of heat radiation by the gases are negligible.

Because of the high concentration of reactive gases, the flow and energy equations are coupled to the species balance equations which, for the $i^{\text {th }}$ species in a $N$-component system are presented in Appendix B. The last term in equation (B.4), $S_{2}$, represents the formation and consumption of the $i^{\text {th }}$ species by gas-phase reactions (homogeneous reactions). We have assumed no homogeneous reaction $\left(S_{\imath}=0\right)$. For tungsten deposition we have assumed that the deposition process is fully determined by surface chemistry on the heated substrate (heterogeneous reaction which will be described as a boundary condition). The number of species $(N)$ is $3\left(\mathrm{WF}_{6}(1), \mathrm{HF}(2)\right.$ and $\left.\mathrm{H}_{2}(3)\right)$.

When the heat, and mass transport processes are coupled, as in multi-component and/or non-isothermal diffusion (commonly encountered in film growth), then the simple phenomenological Fick's law is extended [25]. Multicomponent effects are minor in dilute mixtures and are accurately described with the binary form of Fick's law. Multicomponent diffusion is most simply described by generalizing the binary diffusion to a $N$-component system [26]. In equation (B.6) the $D_{23}$ are multicomponent diffusion coefficients.

By defining thermal diffusion coefficient $D_{2}^{T}$ related to the Soret coefficients, the thermodiffusion flux is described by equation (B.7) in Appendix B. Kleijn [9] has proposed different expressions for the multi-component thermal diffusion coefficients based on the kinetic theory. He pointed out that in gas mixtures containing significant amounts of both heavy and light gas molecules (like $\mathrm{WF}_{6}$ and $\mathrm{H}_{2}$ ), the accurate evaluation of thermal diffusion coefficient may 
be important and approximations may lead to large discrepancies. So, we have calculated the more accurate values of $D_{\imath}^{T}$ as proposed and described in reference [9].

The transport coefficients $\left(\mu, \lambda, C_{p}\right)$ of each gas species and the binary ordinary diffusion coefficients were calculated from Chapman-Enskoog kinetic theory, assuming Lennard-Jones [6-12] interaction potentials [27]. The common rules were used to calculate the transport coefficients of the gas mixture as a function of pressure, temperature and gas concentration [24].

3.2. Boundary Conditions. - Boundary conditions must be specified for the velocity vector, temperature and concentrations of species:

- non-slip conditions: $u=v=0$ at the bounding walls $\left(T_{\text {gas }}=T_{\text {wall }}\right)$,

- isothermal side wall and induction coil: $T=300 \mathrm{~K}$,

- specified inlet temperature $(300 \mathrm{~K})$ and substrate temperature,

- specified species concentration in the inflow,

- zero species diffusion on non-reacting walls.

On the substrate, the $\mathrm{H}_{2}$ reduction of $\mathrm{WF}_{6}$ takes place; the surface reaction rate $R_{\mathrm{s}}$ (in $\mathrm{kg} \mathrm{m}^{-2} \mathrm{~s}^{-1}$ ) was deduced from equation (2). The deposition rate $G$ of tungsten was calculated from:

$$
G=R_{\mathrm{s}} \frac{m_{\mathrm{w}}}{\rho_{\mathrm{w}} m_{1}}
$$

For a very fast chemical reaction, the mass-transport limited regime is reached and the deposition rate approaches the maximum diffusive flux on the substrate surface. The boundary conditions are on the substrate:

$$
\begin{aligned}
& w_{1}=0 \\
& \mathbf{n} \cdot\left(\rho w_{1} \mathbf{v}+\mathbf{J}_{1}+\mathbf{J}_{1}^{T}\right)=-\frac{1}{6} \mathbf{n} \cdot\left(\rho w_{2} \mathbf{v}+\mathbf{J}_{2}+\mathbf{J}_{2}^{T}\right)
\end{aligned}
$$

The deposition rate of tungsten $G$ was calculated from:

$$
G=\mathbf{n} \cdot\left(\rho w_{1} \mathbf{v}+\mathbf{J}_{1}+\mathbf{J}_{1}^{T}\right) \frac{m_{\mathrm{w}}}{\rho_{\mathrm{w}} m_{1}}
$$

The series of governing equations were numerically solved in two-dimensional, axisymmetric form by the finite element method using Flux-Expert simulator [28, 29]. For most calculations, about 2000 grid points were used. The grid independence was checked using more grid points (2000-5000). The deposition rate variations were less than $2 \%$. The calculations were carried out on a SUN sparcstation 2. To reach the convergence on the standard grid for the resolution of the coupled equations, about 6 hours cpu were needed.

3.3. General Features of the Gas Flow. - Some examples of the distribution of the gas flow velocity in the CVD reactor are shown in Figure 3. Some recirculation flow pattern region caused by the small inlet tube can be identified at the entrance. In the $773 \mathrm{~K}$ - $1073 \mathrm{~K}$ temperature range, for the lowest pressure used $\left(P=4 \times 10^{3} \mathrm{~Pa}\right)$, for an inlet flow of $200 \mathrm{sccm}\left(8 \mathrm{sccm} \mathrm{WF}_{6}\right.$ ) a perfect laminar flow was established near the substrate. At higher pressures $\left(P=10^{4} \mathrm{~Pa}\right)$, buoyancy-driven flows, caused by thermal and solutal gradients, occur over and under the substrate (Fig. 3b). The temperature contours are shown in Figure 4. Figure 5 illustrates the consumption of $\mathrm{WF}_{6}$ from the entrance to the substrate. $W_{6}$ enters the reactor with a specified molar fraction of $4 \%$ and is totally consumed on the substrate (mass-transport limited regime). It has been already shown that thermodiffusion is of importance for the establishment of the concentration field [10-12]. Others results concerning the flow, temperature and concentration fields can be found in reference [10-12]. 


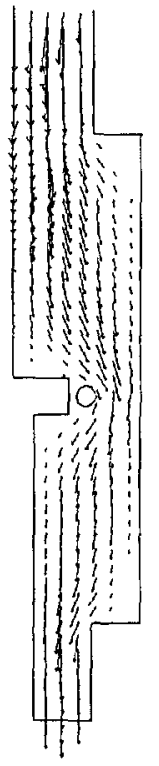

a)

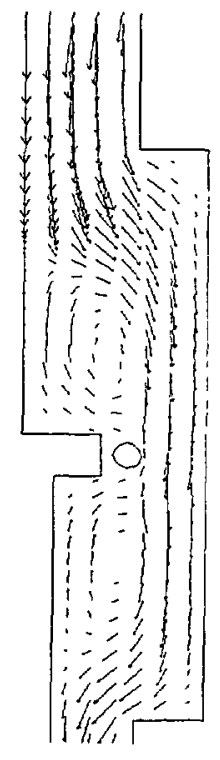

b)

Fig. 3. - Model predictions of the velocity field features for an inlet flow (STP) of $200 \mathrm{sccm}, 8 \mathrm{sccm}$ $W_{6}(4 \%)$ and a substrate temperature of $1073 \mathrm{~K}$. a) $P=4 \times 10^{3} \mathrm{~Pa}$; b) $P=10^{4} \mathrm{~Pa}$ (enlarged view).

\section{Comparison of Numerical Predictions with Experimental Results}

Figure 6 shows an excellent agreement between the temperature dependence of the averaged deposition rate, including the transition point where the reaction becomes mass-transport limited, about $773 \mathrm{~K}$, for a pressure of $4 \times 10^{3} \mathrm{~Pa}$, a total flow rate of $200 \mathrm{sccm}$ and a WF molar fraction of $4 \%(8 \mathrm{sccm})$. The apparent activation energy, $E$ (Eq. $(2))$ in the chemical reaction controlled regime (for temperatures lower than $773 \mathrm{~K}$ for the experimental conditions of Fig. 6), is about $65 \mathrm{~kJ} \mathrm{~mol}^{-1}$ The literature data are ranging from 60 to $70 \mathrm{~kJ} \mathrm{~mol}^{-1}[1-17]$. When the pressure is lowered or when the amount of $W_{6}$ is increased, the transition point is moved towards a higher temperature (about $860 \mathrm{~K}$ for a total pressure of $1000 \mathrm{~Pa}$, a total flow rate of $200 \mathrm{sccm}$ and $\mathrm{a}_{\mathrm{WF}}$ molar fraction of $8.5 \%$ ). More details concerning Arrhenius diagrams for tungsten deposition can be found in reference [4]. The experimental and predicted deposition rates are average values because the coating thickness over the cylindrical substrate is not uniform. Its shape is rather complex compared to that of the silicon wafers used in the previous modelling studies [9-17]. The uniformity of the coating thickness will be discussed further.

Figure 7 shows the dependence of the deposition rate on the hydrogen partial pressure for a fixed temperature $(1073 \mathrm{~K})$ and a fixed amount of $\mathrm{WF}_{6}(160 \mathrm{~Pa})$. At low $\mathrm{H}_{2}$ partial pressures $\left(<1500 \mathrm{~Pa}\right.$ ), the rate increases with increasing $\mathrm{H}_{2}$ pressure. The deposition process is in the chemical reaction controlled regime. Then, at higher $\mathrm{H}_{2}$ partial pressures the rate decreases as the $\mathrm{H}_{2}$ partial pressure increases for a fixed $\mathrm{WF}_{6}$ flow. The deposition rate becomes mass transport controlled. The features of the modelling results are similar to those of the experimental results. Therefore, the model always gives higher average deposition rates. In the kinetically controlled regime, this is mainly caused by the fitting parameter $E$, which 


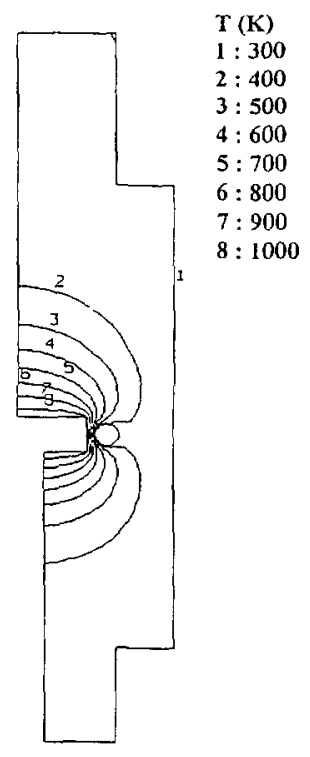

Fig. 4

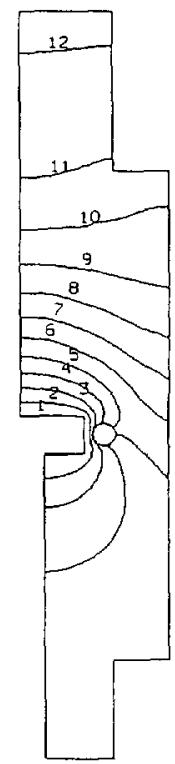

Fig. 5

Fig. 4. - Temperature contours inside the CVD reactor for the conditions of Figure 3a.

Fig. 5. - Concentration contours of $\mathrm{WF}_{6}(\mathrm{~mol} \%)$ inside the CVD reactor for the conditions of Figure 3a) (line 1 = 0.363\%; line $12=3.63 \%$; equirepartition between line 1 and 12 ).

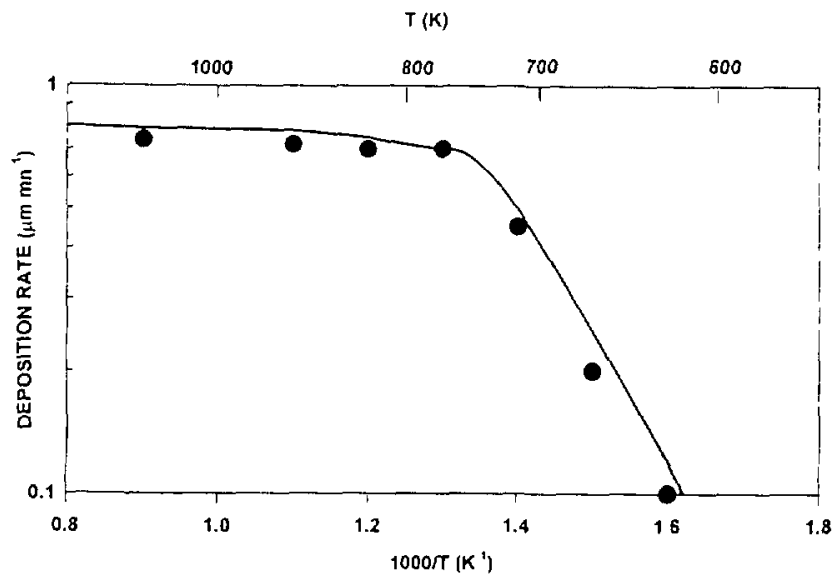

Fig. 6. - Arrhenius plot for a total pressure of $4 \times 10^{3} \mathrm{~Pa}$, an inlet flow of $200 \mathrm{sccm}(8 \mathrm{sccm} \mathrm{WF} 6$ $(4 \%))$. (-) Modelling results; $(\bullet)$ experimental points.

have been averaged within the investigated experimental range. In the transport controlled region, the model assumes an infinite rate of reaction, translated by a zero boundary condition for $\mathrm{WF}_{6}$ mass fraction. A near-zero mass fraction would lower the deposition rate. An other 


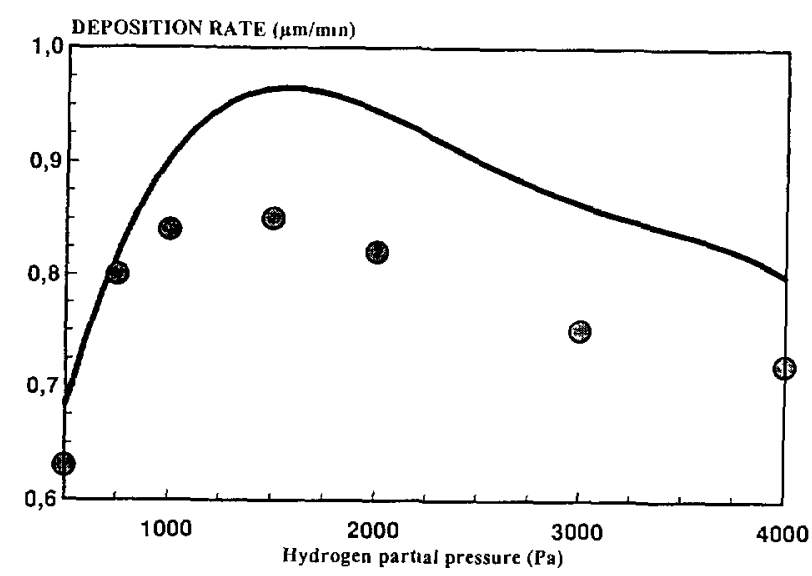

Fig. 7. - Dependence of the deposition rate on hydrogen partial pressure for a fixed temperature $(1073 \mathrm{~K})$, a fixed partial pressure of $\mathrm{WF}_{6}(160 \mathrm{~Pa})$ and a total flow rate of $200 \mathrm{sccm}$. (-) Modelling results $;(\bullet)$ experimental points.

source of deviation would be the evolution of the transport coefficients with the temperature and concentration of the gas mixture. This rapid discussion about the uncertainties associated with the boundary conditions on rather complex reactive areas and with transport coefficients evidences that a sensitivity analysis would be performed.

The results presented above concerned average deposition rates and their comparison with average model predictions owing to the shape of the substrate and to the thickness variations. They have clearly shown the transition between kinetic and mass transport limited regimes. It is interesting to note that the trends obtained are similar to those obtained by Mc Inerney et al. [7] dealing with high pressure CVD tungsten at lower temperatures $(<700 \mathrm{~K})$. With these averaged results, we think that the models proposed for the high temperature deposition of tungsten can be applied to different reactor geometry.

Within the range of the operating parameters explored, the growth rate is sometimes nonuniform over the substrate. The flux of the depositing species is proportional to both the concentration gradient due to diffusive and convective effects and to the deposition area. We have shown that the mass-transport limited regime occurs at temperature higher than $773 \mathrm{~K}$ for a total flow rate of $200 \mathrm{sccm}$, a total pressure of $4000 \mathrm{~Pa}$ and a $\mathrm{H}_{2}-4 \% \mathrm{WF}_{6}$ mixture. As a base case, we have chosen these process conditions within this regime to discuss about the spatial non-uniformity of the deposition. Figure 8 depicts the variations of the deposition rate at $1073 \mathrm{~K}$. These results, confirmed experimentally, can be explained by convective effects at the substrate edges and near the induction coil and the rapid depletion of $\mathrm{WF}_{6}$ along the substrate. Figure 9 depicts more clearly the uniformity of the coating on the top surface of the substrate. When the pressure is lowered and when the concentration of $W_{6}$ is increased up to $8 \%$, the uniformity is improved. The model over-predicts the variations at the edge of the substrate; this is mainly due to the abrupt changes in boundary conditions within the model. In spite of these discrepancies, there is a good agreement between the trends obtained by modelling and experimental results. More refined diagnostics will be now presented in order to control the accuracy of the proposed model rather than to discuss about the transport phenomena leading to tungsten deposition. 


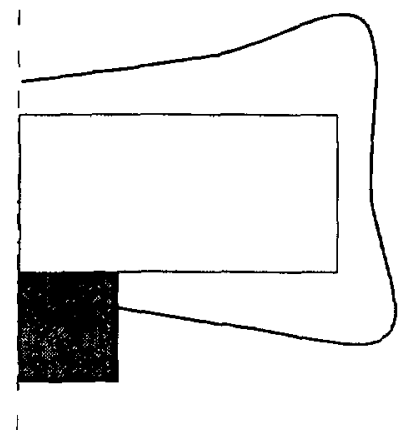

Fig. 8. - Schematic representation of the uniformity of the coating for an inlet flow of $200 \mathrm{sccm}, 8$ sccm $W_{6}(4 \%)$, a total pressure of $4 \times 10^{3} \mathrm{~Pa}$ and a substrate temperature of $1073 \mathrm{~K}$ (only the right part of the substrat is shown).

Table II. - Comparison between measured and predicted values assuming that the deposition process is mass transport contralled.

\begin{tabular}{|c|c|c|c|c|}
\hline \multicolumn{5}{|c|}{ Total flowrate $=200 \mathrm{sccm}(\mathrm{STP}) ;$ Total pressure $=4 \mathrm{hPa}, W F_{6}$ mol fraction $=4 \%$} \\
\hline $\begin{array}{l}\text { Distance trom substrate } \\
(\mathrm{mm})\end{array}$ & $\begin{array}{l}\text { Averuged measured } \\
\text { temperature }(\mathrm{K})\end{array}$ & $\begin{array}{l}\text { Computed temperature } \\
(\mathrm{K})\end{array}$ & $\begin{array}{l}\text { WF } 6 \text { mol fraction measured } \\
\qquad\left(\times 10^{2}\right)\end{array}$ & $\begin{array}{l}\text { WF }_{6} \text { mal fiaction computed } \\
\qquad\left(\times 10^{2}\right)\end{array}$ \\
\hline \multicolumn{5}{|c|}{$\mathrm{T}=773 \mathrm{~K}$} \\
\hline 25 & 745 & 728 & 014 & 015 \\
\hline 5 & 712 & 685 & 025 & 019 \\
\hline \multicolumn{5}{|c|}{$T=923 \mathrm{~K}$} \\
\hline 25 & 843 & 867 & 013 & 012 \\
\hline 5 & 820 & 808 & 0185 & 014 \\
\hline \multicolumn{5}{|c|}{$T=1073 \mathrm{~K}$} \\
\hline 25 & 995 & 1002 & 014 & 010 \\
\hline 5 & 978 & 032 & 019 & 1) 17 \\
\hline
\end{tabular}

Previous results have compared a model involving coupled phenomena and the main results of the reactive process: the deposition rate. More specific diagnostics are necessary to improve the validation of the software package developed. With the Raman equipment described previously, we have measured temperature and concentrations of the reactive species in the gas phase over the substrate during the deposition process. For a fixed total flow of $200 \mathrm{sccm}$ and different substrate temperatures, pressures and $\mathrm{WF}_{6}$ flow rates, temperature and $\mathrm{WF}_{6}$ concentration were measured and computed from the substrate $(z=0)$ to $5 \mathrm{~mm}$ above the substrate along the symmetry axis. Tables II and III show the two sets of results. They have been obtained by two quite different approaches and without any fitting parameters. They are presented without any sensitivity studies as raw data. For the modelling approach, the transport coefficients were calculated from the gas kinetic theory and the boundary conditions fitted with averaged deposition rates. For the Raman approach, the results are the average values of a series of experiments. It is not possible, at present time, to discuss about the discrepancies between the two sets of results. They are inherent in the methods. It is only possible to point out that the results are of the same order of magnitude and that each approach gives consistent results. These preliminary results are however encouraging and contribute to demonstrate that the modelling approach is a potential tool for tungsten CVD knowledge and process optimization and that the Raman equipment is able to record data within the gas 


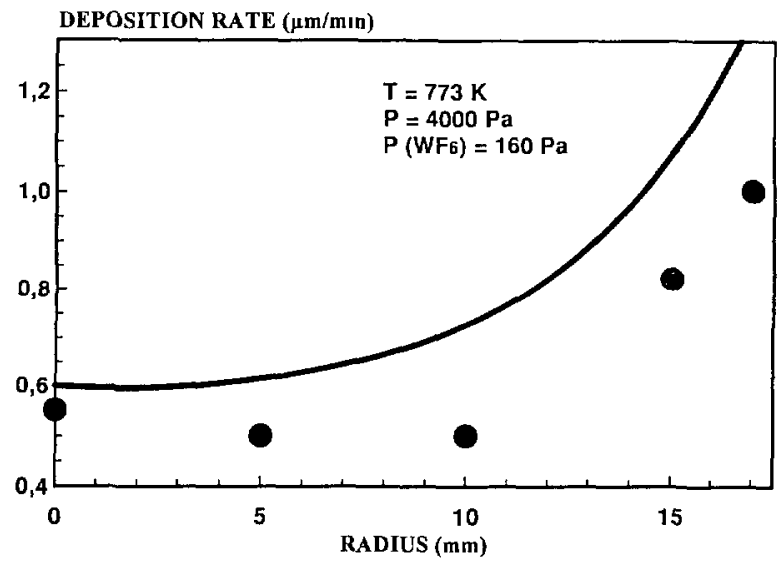

a)

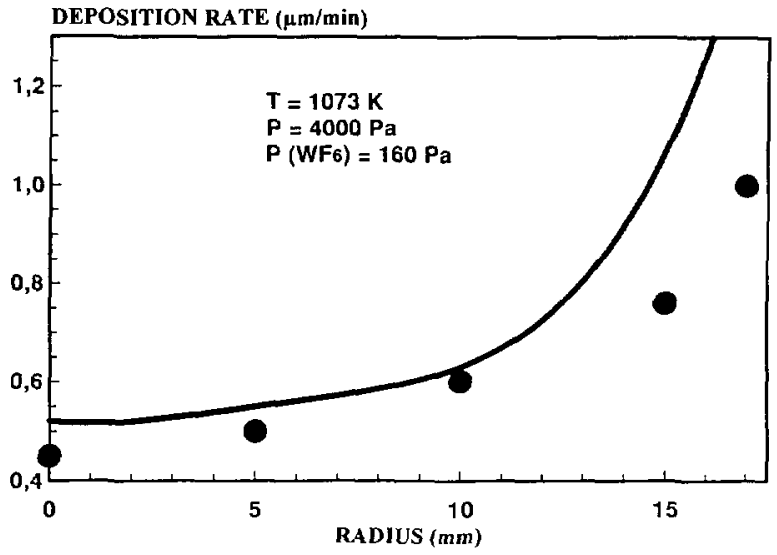

b)

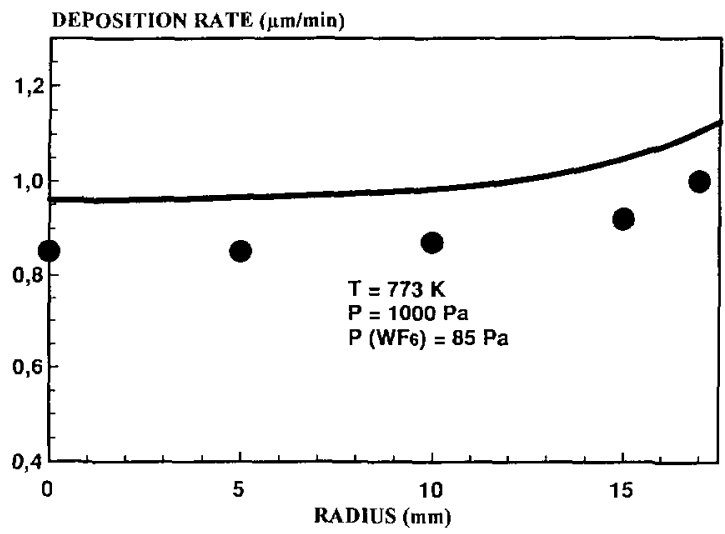

c)

Fig. 9. - Radial uniformity of the top surface of the coating for: a) $P=4 \times 10^{3} \mathrm{~Pa} ; T=773 \mathrm{~K} ; 4 \%$ $\mathrm{WF}_{6}$ b) $P=4 \times 10^{3} \mathrm{~Pa} ; T=1073 \mathrm{~K} ; 4 \% \mathrm{WF}_{6} ;$ c) $P=10^{3} \mathrm{~Pa} ; T=773 \mathrm{~K} ; 8.5 \% \mathrm{WF}_{6}$; $(\longrightarrow$ ) modelling results; $(\bullet)$ experimental points. 
Table III. - Comparison between measured and predicted values assuming that the deposition process is kinetically controlled.

\begin{tabular}{|c|c|c|c|c|}
\hline \multicolumn{5}{|c|}{ Total flow rute $=200 \mathrm{sccm}$ (STP), Total pressure $=1 \mathrm{kPa}, \mathrm{WFF}_{6}$ mol fraction $=8,5 \%$} \\
\hline $\begin{array}{c}\text { Distance from substrate } \\
(\mathrm{mm})\end{array}$ & $\begin{array}{c}\text { Averaged measured } \\
\text { temperature }(\mathrm{K})\end{array}$ & $\begin{array}{c}\text { Computed temperature } \\
(\mathrm{K})\end{array}$ & $\begin{array}{c}\mathrm{WF}_{6} \text { mol fraction measured } \\
\left(\mathrm{x} 10^{2}\right)\end{array}$ & $\begin{array}{c}\text { WF } \\
\text { mol traction computed } \\
\left(\times 10^{2}\right)\end{array}$ \\
\hline 2.5 & 750 & 730 & 344 & 335 \\
5 & 720 & 688 & 38 & 337 \\
\hline
\end{tabular}

phase during the deposition process. However, only one flow rate has been studied. As we have shown in Figure 3, convective effects can modify the flow features and consequently the temperature and concentration fields. Further studies taking into account a wider range of process parameters should be performed. Within the limits of the investigated experimental range, numerical modelling should now be applied to an industrial configuration to check the influence of the geometry and the influence of process parameters on the deposition rate on complex shaped substrates. It would be possible to delimit the geometric artefacts from the changes in deposition kinetics.

\section{Conclusion}

A two-dimensional model has been developed for the calculation of reactive mass transport occurring during the deposition of thick tungsten coatings at high temperatures $(773-1073 \mathrm{~K})$ in a reactor resulting from a scaled model of an industrial one. The agreement between the computed and experimental results obtained from microscopic examinations and from in-situ Raman measurements demonstrates the validity of the proposed model and its predictive capabilities. We have investigated a temperature range little studied owing to the importance of tungsten CVD processed at temperatures lower than $773 \mathrm{~K}$ for metallization problems in microelectronics. The physical and chemical phenomena involved during the high temperature deposition process are similar to those observed at lower temperatures. Finally, we have shown that the temperatures and the $\mathrm{WF}_{6}$ partial pressures can be recorded by a Raman equipment during the deposition process.

\section{Appendix A}

\section{Notations}

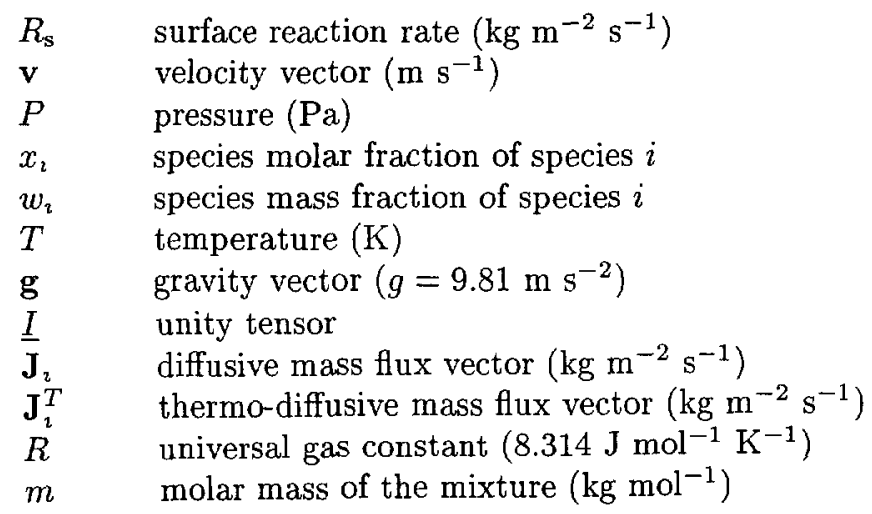




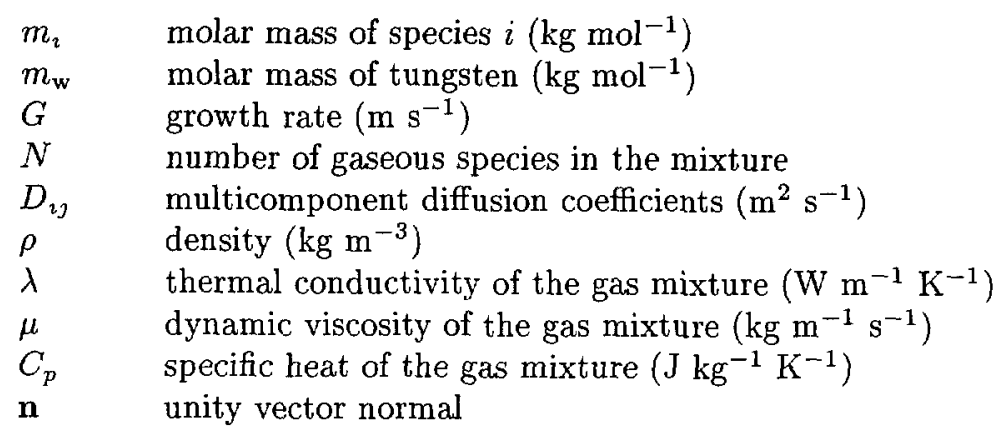

\section{Appendix B}

\section{General equations}

$$
\begin{aligned}
& \nabla \cdot(\rho \mathbf{v})=0 \\
& \nabla \cdot(\rho \mathbf{v} \mathbf{v})=\nabla \cdot\left(\mu\left(\nabla \mathbf{v}+(\nabla \mathbf{v})^{t}\right)-\frac{2}{3}(\nabla \cdot \mathbf{v}) \cdot \underline{I}\right)-\nabla P+\rho \mathbf{g} \\
& C_{p} \nabla \cdot(\rho \mathbf{v} T)=\nabla \cdot(\lambda \nabla T) \\
& \nabla \cdot\left(\rho \mathbf{v} w_{\imath}\right)=-\nabla\left(\mathbf{J}_{\imath}+\mathbf{J}_{\imath}^{T}\right)+S_{\imath} \quad i=1 \ldots N-1 \\
& \sum_{\imath=1}^{N} w_{\imath}=1 \\
& -\mathbf{J}_{\imath}=\sum_{J=1}^{N-1} \rho D_{\imath \jmath} \nabla \mathbf{w}_{\jmath} \\
& -\mathbf{J}_{\imath}^{T}=D_{\imath}^{T} \frac{\nabla T}{T}
\end{aligned}
$$

\section{References}

[1] Broadbent E.K. and Ramiller C.L., Selectrve low Pressure Chemical Vapor Deposition of Tungsten, J. Electrochem. Soc, 131 (1984) 1427-1433.

[2] Mc Conica C.M. and Krishnamani K., The Kinetics of LPCVD Tungsten Deposition in a Single Wafer Reactor, J. Electrochem. Soc. 133 (1986) 2542-2548.

[3] Pauleau Y. and Lami Ph., Kinetics and Mechanism of Selective Tungsten Deposition by LPCVD, J. Electrochem. Soc. 132 (1985) 2779-2784.

[4] Auvert G., Pauleau Y. and Tonneau D., Growth Kinetics of 'Tungsten Microstructures Produced via The Hydrogen Reduction of Tungsten Hexafluoride on laser heated substrates, J. Appl. Phys. 71 (1992) 4533-4543.

[5] Creighton R. and Parmeter J.E., Metal CVD for Microelectronic Applications: an Examination of Surface Chemistry and Kinetics, Crit. Rev. Soled State Mat. Sci. 18 (1993) 175-238.

[6] Joshi R.V., Mehter E., Chow M., Ishaq M., Kang S., Geraghty P. and Mc Inerney J., High Growth Rate CVD-W Process for Filling High Aspect Ratio Sub-micron Contacts Lines, in Tungsten and other Advanced Metals for VLSI/ULSI Applications V, Wong S.S. and Furukawa S., Eds. (The Materials Research Society, Pittsburg, 1990) pp. 157-166.

[7] Mc Inerney E.S., Chin B.L. and Broadbent E.K., The Rate Kinetics of High Pressure CVD Tungsten, in Tungsten and other Advanced Materials for VLSI Applications, Smith G.C. and Blumenthal R. Eds, The Materials Research Society (Pittsburg, PA, 1991) pp. 249-256. 
[8] Gaillard D., CVD : Des Revêtements Submicroniques à l'Obtention de Pièces Massives, Mat. Tech. 3 (1989) 1-16.

[9] Kleijn C.R. and Werner C., Modelling of Chemical Vapor Deposition of Tungsten Films, in Progress in Numerical Simulation for Microelectronics, Vol. 2, K. Merten and A. Gilg Eds. (Birkhaüser Verlag Basel, 1993) pp. 83-136.

[10] Kleijn C.R., Hasper A., Holleman J., Hoogendoorn C.J. and Middelhoek J., Transport Phenomena in tungsten LPCVD in a single-wafer reactor, J. Electrochem. Soc. 138 (1991) 509-517.

[11] Arora R. and Pollard R., A mathematical model for Chemical Vapor Deposition Influenced by Surface Reaction Kinetics: Application to low Pressure Deposition of Tungsten, J. Electrochem. Soc. 138 (1991) 1523-1537.

[12] Ulacia J.J., Howell S., Körner H. and Werner C., Flow and Reaction Simulation of a Tungsten CVD Reactor, Appl. Surf. Scr. 31 (1989) 370-385.

[13] Chang J.C. and Megna L.A., Heat Flow Modeling of Chemical Vapor Deposition of Tungsten for Improved Film Thickness Uniformity, J. Elec. Mat. 21 (1992) 1019-1025.

[14] Jasinski J.J. and Kang S.S., Application of Numerical Modeling for CVD Simulation. Test case: Blanket Tungsten Deposition Uniformity, in Tungsten and Other Advanced Metals for VLSI Applications, Smith G.C. and Blumenthal R., Eds (The Materials Research Society, Pittsburgh, 1991) pp. 219-230.

[15] Werner C., Ulacia J., Hoffmann C. and Flynn P., Equipment Simulation of Selective Tungsten Deposition, J. Electrochem. Soc. 139 (1992) 566-574.

[16] Rode J.E. and Schmitz J.E.J.,Study of Reactor Design by Computational Fluide Dynamics, in Advanced Metallization for ULSI Applications (Formerly Workshop in Tungsten and Other Advanced Metals for ULSI Applications, October 8-10 1991, Murray Hill NJ, USA), Rana V.S., Joshi R.V. and I. Ohdomari, Eds. (Materials Research Society, Pittsburgh, 1992) pp. 105-111.

[17] Fotiadis D.I., Boekholt M., Jensen K.F. and Richter W., Flow and Heat Transfer in CVD Reactors: Comparison of Raman Temperature Measurements and Finite Element Model Predictions, J. Cryst. Growth 100 (1990) 577-599.

[18] Devonshire R., Coherent anti-Stockes Raman Scattering (CARS) in CVD, Chemtronics 2 (1987) 183-198.

[19] Gaufrès R., Huguet P., Boya D. and Lafforêt J., Montage Raman de hautes performances adapté à la caractérisation de la phase gazeuse dans des opérations de dépôt chimique en phase vapeur: Résultats préliminaires", J. Phys. France C5 (1989) 855-860.

[20] Huguet P., Caractérisation d'une phase gazeuse en cours de réaction par Raman spontané, Thesis (Montpellier, France, 1992).

[21] Gaufrès R. and Sportouch S., Détermination des constantes de rotation $\alpha$ à partir des contours des branches $\mathrm{Q}$ des bandes Raman. Cas des vibrations symétriques des molécules linéaires, C. $R$. Acad. Scı. Parıs 272 (1971) 995-998.

[22] Gaufrès R. and Sportouch S.,Contours des bandes dans les spectres Raman de gaz, in Advanced in Raman Spectroscopy, Mathieu, J.-P. Ed. (Heyden London, 1973) pp. 478-492.

[23] Gaufrès R., Analysis of Raman contours in vibration-rotation spectra, in Laser Raman Gas Diagnostics, Lapp, M. and Penney, C.M. Eds. (Plenum Press New-York, 1990) pp. 15-43.

[24] Huguet P. and Gaufres R., (1995) Determination of the Temperature of a Gas by a Simple and Accurate Raman Method, J. Raman Spectrosc. (1995) in press.

[25] Bird R.B., Stewart W.E. and Lighfoot E.N., Transport Phenomena (John Wiley, New York, USA, 1960).

[26] Cussler E.L., Diffusion, Mass transfer in fluid systems, Cambridge University Press (1984).

[27] Hirschfelder J.O., Curtiss C.F. and Bird R.B., Molecular Theory of Gases and Liquids (John Wiley, New York, USA, 1967).

[28] Flux-Expert Software Package Guide, DT2I, 38240 Meylan, France (1993).

[29] Pons M., Arena C. and Klein R., Modelling of Cold Wall Chemical Vapor Deposition Reactors, J. Phys. France C5 (1989) 57-65. 